\title{
Poor survival on an artificial diet of two genotypes of the aphid Myzus persicae: a fitness cost of insecticide resistance?
}

Article

Accepted Version

van Emden, H. F., Foster, S. P. and Field, L. M. (2014) Poor survival on an artificial diet of two genotypes of the aphid Myzus persicae: a fitness cost of insecticide resistance? Agricultural and Forest Entomology, 16 (4). pp. 335-340. ISSN 1461-9563 doi: https://doi.org/10.1111/afe.12063 Available at https://centaur.reading.ac.uk/38055/

It is advisable to refer to the publisher's version if you intend to cite from the work. See Guidance on citing.

To link to this article DOI: http://dx.doi.org/10.1111/afe.12063

Publisher: Blackwells

All outputs in CentAUR are protected by Intellectual Property Rights law, including copyright law. Copyright and IPR is retained by the creators or other copyright holders. Terms and conditions for use of this material are defined in the End User Agreement.

www.reading.ac.uk/centaur 
Central Archive at the University of Reading

Reading's research outputs online 
Poor survival on an artificial diet of two genotypes of the aphid Myzus persicae: a fitness cost of insecticide resistance?

\section{Helmut F. van Emden*, Stephen P. Foster ${ }^{\dagger}$ and Lin M. Field ${ }^{\dagger}$}

School of Biological Sciences, University of Reading, Reading, Berks., RG6 6AS,

†Department of Biological Chemistry and Crop Protection, Rothamsted Research, Harpenden, Herts., AL5 2JQ, UK

Running head: Survival of insecticide resistant Myzus persicae on artificial diet

\section{Keywords}

aphids, artificial diet, carboxylesterase, fitness, genotypes

\section{Correspondence}

Helmut van Emden (corresponding author), School of Biological Sciences, University of Reading, Reading, Berks., RG6 6AS, UK. Tel: +44 (0)118 3788493, Fax +44 (0)118 9352421, email: h.f.vanemden@ reading.ac.uk 


\section{Abstract}

1. A fully susceptible genotype (4106A) of Myzus persicae survived the longest on artificial diet and, in several of the 8 replicates, monitoring was terminated while the culture was still thriving. A genotype with elevated carboxylesterase FE4 at the $\mathrm{R}_{3}$ level (800F) had a mean survival of only 98.13 days and 794J, which combines $\mathrm{R}_{3} \mathrm{E} 4$ carboxylesterase with target-site resistance (kdr), survived for the even shorter mean time of 84.38 days .

2. The poorer survival of the two genotypes with extremely elevated carboxylesteraseresistance was not due to a reluctance to transfer to new diet at each diet change.

3. Although available for only two replicates, a revertant clone of 794J (794Jrev), which has the same genotype as 794J but the amplified E4 genes are not expressed leading to a fully susceptible phenotype, did not appear to survive any better than this clone. This suggests that the poor survival on artificial diet of the extreme-carboxylesterase genotypes is not due to the cost of over-producing the enzyme.

4. That the frequency of insecticide-resistant genotypes is low in the population until insecticide is applied indicates they have reduced fitness, but not necessarily that this reflects a direct cost of expressing the resistance mechanism.

\section{Introduction}

It is usually assumed that insect genotypes that are resistant to insecticides arise at a low frequency in populations, and only become established when selected by repeated insecticide application. This is thought to be because mechanisms of resistance come at a cost to fitness in the absence of insecticide (Crow, 1957; Kliot \& Ghanim, 2012). It is therefore surprising, given the increasing importance of the insecticide resistance problem over the last 60 years and the research directed at managing it, how infrequently any such fitness cost of resistance 
has been demonstrated for either metabolic resistance (Castañeda et al., 2011) or mutations of target site proteins. However, in aphids it has been suggested that, although there is a fitness cost for resistance, it is subtle and difficult to detect, only becoming apparent when the insects are stressed in some way (e.g. Foster et al., 1996, 1997) and not detectable when they are tested under optimal conditions in the laboratory. These papers describe fitness costs imposed on Myzus persicae (Sulzer) (Hemiptera: Aphididae) resistant to insecticides associated with high levels of carboxylesterases (a metabolic mechanism conferring resistance to organophosphates) or a combination of carboxylesterase production with knockdown resistance (kdr) (a target site mechanism conferring resistance to pyrethroids).

Resistant aphids suffered greater mortality in the adverse weather conditions of winter (Foster et al., 1996), a reduced tendency to move from deteriorating leaves to younger leaves (Foster et al., 1996, 1997) and greater mummification by parasitoid wasps (Foster et al., 2007). It has also been shown in laboratory experiments that genotypes of $M$. persicae with MACE resistance (a target site mechanism conferring resistance to pirimicarb) and $\mathrm{R}_{3}$ carboxylesterase resistance (Foster et al., 2000) show reduced reproduction compared with susceptible forms, as also shown for insecticide-resistant genotypes of Schizaphis graminum (Rondani) (Hemiptera: Aphididae) (Rider \& Wilde, 1998; Stone et al., 2000). However, it is arguable that again these results were obtained under stress, especially when (as in the case of M. persicae) excised leaf material was used (van Emden, 1977). However, for Phorodon humuli (Schrank) (Hemiptera: Aphididae), results have either been inconclusive (Hampson \& Madge, 1986, 1987) or a resistant genotype has actually shown both a higher reproductive rate and total fecundity than a susceptible one, although it should be noted that this was a comparison between two genotypes (Lorriman \& Llewellyn, 1983).

The fully-defined artificial diet used at Reading for the continuous culture of $M$. persicae (van Emden, 2009) involves the stress of suboptimal nutrition, since this aphid shows 
extended development, smaller size and much reduced fecundity on diet than when plantreared (van Emden, 1988). Wool \& van Emden (1981) studied esterase variation (using gel polyacrylamide electrophoresis) in 64 cultures of $M$. persicae derived from single founder individuals of 12 genotypes sourced from five different UK research institutions (the Natural History Museum, Rothamsted Research, Wye College, Syngenta Jealott's Hill Research Station and the University of Reading). The cultures were kept on an artificial diet and maintained as described by van Emden (2009) because this provided a simple way of keeping the large number of cultures in a limited space with no likelihood of contamination between them. At the end of their experiments, Wool \& van Emden (1981) reported considerable variation in the survival on diet of the 64 cultures. Ten died out during the second generation, 29 during the third, 18 during the fourth and two during the fifth. In 1980, when the paper was written, the remaining five cultures had reached the tenth generation, and one culture finally only died out after 32 years (van Emden, 2009).

Although there was no replication, it appeared a posteriori that most of the cultures died out before or during generation 3, including all the 35 cultures with high carboxylesterase activity associated with resistance to organophosphate insecticides (Needham \& Sawicki, 1971). In contrast, seven of the 29 cultures with low levels of carboxylesterase survived beyond generation 3 (Fig. 1). We have re-analysed the data using a $\chi^{2}$ association test. The frequency of cultures dying out before and after the third generation differs significantly $(P<$ 0.01) between high and low carboxylesterase cultures, the former cultures showing the higher frequency. However, Wool \& van Emden (1981) probably had several repeats among their higher carboxylesterase genotypes of UK origin, since Fenton et al. (2010a) has found current UK $M$. persicae populations consist of only a limited number of genotypes. However, it is not known if this was the case over thirty years ago when the original study was done. It should also be noted that all seven of the cultures that survived beyond generation 3 (with one 
surviving for over thirty years) were derived from a single source, a University of Reading glasshouse where $M$. persicae had become naturally established in 1962 and, when tested by ADAS at their Reading laboratory, had been found to have an $\mathrm{LD}_{50}$ to the organophosphate insecticide malathion 4000-fold lower than any population from commercial glasshouses in southern England (H.J. Gould, unpublished ADAS bioassay results).

By contrast with the a posteriori speculation of Wool \& van Emden (1981), the present study was a replicated a priori test of whether three genotypes with different known levels of carboxylesterase-based resistance would differ in their survival time on artificial diet. Additionally, it was of interest whether their survival time would inversely correspond with their level of carboxylesterase resistance. Three genotypes of $M$. persicae of known insecticide resistance status were selected from the the long term cultures at Rothamsted Research reared on Chinese cabbage, Brassica napus L. V. chinensis CV. Wong Bok. Clones 4106A and 794J were both originally collected from potato crops. 800F was collected from peach. Some work (Eggers-Schumacher, 1983) has been done on the suitability of different host plants for insecticide-susceptible and-resistant $M$. persicae clones. He showed that an advantage was gained by the esterase-S aphids through a greater readiness to transfer to and reproduce on less suitable hosts such as chrysanthemum, broad bean and spinach beet.

\section{Materials and methods}

Of the three genotypes of $M$. persicae chosen for study, 4106A is classed as fully insecticidesusceptible (S), 800F shows elevated carboxylesterase FE4 production at what is classified as the $\mathrm{R}_{3}$ (extreme) level (conferring strong resistance to organophosphates), and 794J combines $\mathrm{R}_{3} \mathrm{E} 4$ carboxylesterase with $\mathrm{kdr}$ in the homozygous form (conferring strong target site resistance to pyrethroids). Although $800 \mathrm{~F}$ and $794 \mathrm{~J}$ make slightly different carboxylesterases 
(unlikely to affect the resistance conferred), in both cases the increased enzymes correlate with amplification of the structural genes (E4 or FE4). In the case of E4 in 794J this is associated with a major chromosome abnormality (an A1-3 translocation) not found in 800F where the FE4 genes are located in several clusters around the aphid genome. It is highly unlikely that $794 \mathrm{~J}$ and $800 \mathrm{~F}$ had similar genomes as the former was collected from an asexual UK population and the latter from a sexual population in Italy. 794Jrev was isolated from the 794J clone and therefore, bar any subsequent mutations and the simple change that occurred in gene methylation (associated with gene silencing), will have been genetically identical. 794Jrev has the same amplified E4 genes in the same background genome as 749J but does not express them: thus it has the same genotype but a different, insecticide-susceptible phenotype. All aphid clones were monitored for their resistance profiles using biochemical and DNA diagnostics at Rothamsted to ensure they had not become contaminated. The aphid clones were also reared on excised leaves using a small box cage system developed by Blackman (1971) which has proved to be very resilient to aphid cross contamination over the years. Each time new clone boxes were set up the work surface was cleaned thoroughly between clones. Furthermore, microsatellite DNA analysis, done periodically on some of the aphid clones in culture at Rothamsted, has shown no changes with time.

The artificial diet was based on that developed for M. persicae by Mittler \& Dadd (1962), following the procedures for preparation and usage described by van Emden (2009). Initially, a small excised Chinese cabbage leaf supporting aphids was taken from the Rothamsted cultures to Reading in a small box cage and the aphids transferred to the artificial diet with a fine paint brush. Later the tubes with diet sachets were taken to Rothamsted and the aphids transferred on site.

Due to the method of aphid culture at Rothamsted, only small numbers (6-12) of mixed instars of M. persicae could be started on the artificial diet. However, within $48 \mathrm{~h}$ 
reproduction had increased numbers to 30-50 and all cultures built up to more than it was possible to count before any decline set in. Any inequalities in the initial inoculate were therefore unimportant.

The aphid cultures on the artificial diet were kept in two environments. For the first five replicates, a plant growth room $\left(\min 18, \max 25^{\circ} \mathrm{C}\right.$ ) was available, but the last 3 replicates had to be kept at laboratory temperature $\left(\min 13, \max 27.5^{\circ} \mathrm{C}\right)$. The aphids were provided with sachets of new diet every 2-3 days (usually on each Monday, Wednesday and Friday) and numbers of aphids below 50 were recorded for each genotype until the culture died out. In many of the replicates, numbers of the insecticide-susceptible genotype (4106A) were still large and even increasing when the last other genotype died out, and in these replicates it was considered pointless to continue recording for more than a few extra weeks, since the main interest in the experiment was the order of extinction rather than duration of the genotypes.

Between February 2006 and June 2011, the experiment was repeated eight times for the three main genotypes, and twice for 794Jrev. Unfortunately the culture of this revertant clone at Rothamsted died out after only two replicates had been completed. Therefore, although the data for this clone are presented in Fig. 2 and Table 1, they were not included in any of the statistical analyses. The data for the number of days the cultures survived on artificial diet were subjected to analysis of variance, with the eight dates as independent replicates. However, in order to preserve adequate equality of variances between treatments (Table 1), the maximum datum for 4106A was 14 days longer than that of the last other genotype to extinguish.

The artificial diet technique involves a change to new diet every 2-3 days. This is accomplished by abutting the open ends of the old diet tube and a new one and puncturing the old sachet to allow the diet to run out (van Emden, 2009). Three to four times a week, therefore, the aphids had to climb up the side of the tubes to transfer themselves to new diet. 
As mentioned above, Foster et al. $(1996,1997)$ noticed a reluctance of insecticide-resistant M. persicae to leave deteriorating leaves and this lower mobility, coupled with the regular need of the aphids to transfer to new diet might have accounted for any poorer survival on diet of the carboxylesterase-resistant genotypes. Therefore, the number of aphids still on the old punctured sachet at each diet change was monitored for the first 12 diet changes in the experiments started on 11 February 2010, 23 May 2010 and 1 June 2011, and analysed with 3 replicates by analysis of variance as for the survival data.

\section{Results}

Survival of three M. persicae genotypes on artificial diet

In the analysis of variance for the three genotypes $4106 \mathrm{~A}, 800 \mathrm{~F}$ and $794 \mathrm{~J}$, there was a clear effect of genotype $\left(F_{2,14}=17.02, P<0.001\right)$, with survival of all three genotypes (Table 1$)$ differing from each other ( $\mathrm{LSD}$ at $P=0.05=10.57, P<0.05$ ). The fully susceptible genotype (4106A) consistently performed better (mean 113.12+ days) than the other two, with the

differences being most obvious when survival for more than 14 days longer than that of the other genotypes was included (Fig. 2). In four of the replicates (those starting on 9 May and 6 November 2006, 12 October 2009 and 1 June 2011) there was no sign of 4106A aphid numbers falling when recording was stopped.

Of the two resistant genotypes, survival of 794J (mean 84.38 days) was significantly poorer than that of $800 \mathrm{~F}$ (mean 98.13 days) with $794 \mathrm{~J}$ only surviving longer than $800 \mathrm{~F}$ in one replicate (started on 21 January 2008).

All of the genotypes showed high variation of duration between replicates; in some they all survived for 100-200+ days, in others survival of all was very much lower (Fig. 2). 
Numbers of the three M. persicae genotypes included in the analysis that remained on the old punctured diet sachet after the sachets were changed

The data were again analysed by analysis of variance, but in order to reduce the frequency of low numbers, the data analyzed were the number of aphids which had not moved to the new diet sachet summed over three consecutive diet changes for each of four consecutive periods (of 6-7 days) soon after the cultures had been established (usually after 4-6 days). There was no significant effect of genotype in the analysis $\left(F_{2,33}=0.098, P>0.05\right)$. In order of increasing carboxylesterase resistance, the mean number $\left(\operatorname{LSD}_{P=0.05}=18.03\right)$ of aphids not moving at a diet change was 6.25 (4106A), $7.36(800 \mathrm{~F})$ and $6.42(794 \mathrm{~J})$.

Survival of the revertant clone 794Jrev on artificial diet

As mentioned earlier, the revertant clone was only available for inclusion in two replicates before the culture at Rothamsted died out. There are therefore just two data sets for survival to compare with $794 \mathrm{~J}$.

In the replicate started on 6 November 2006, the survival durations on diet were 179 days for 794J and 189 for 794Jrev; the comparable durations in the replicate started on 21 January 2008 were 138 and 91 days. Thus, although in one replicate the survival of the revertant clone was 10 days longer than that of 794J, it was actually considerably shorter (by 47 days) in the second, suggesting that there are no differences between these two clones. Also, in both replicates survival of 794Jrev was not longer ( 7 and 30 days shorter) than that of the other carboxylesterase-based resistant genotype 800F. 


\section{Discussion}

Our experiments confirmed the report of Wool \& van Emden (1981) that different genotypes of $M$. persicae differ in how long they can be maintained on the fully-defined artificial diet originally developed by Mittler \& Dadd (1962). Although there was large variation in the overall survival on diet between the eight experimental replicates, the order in which the three genotypes died out was statistically separable, with the shortest mean survival in 794J, followed by $800 \mathrm{~F}$ and then $4106 \mathrm{~A}$ (the fully susceptible aphid clone) showing the longest survival. Indeed, in several replicates recording was terminated after the other two genotypes had died out, but while the 4106A culture was still thriving. Indicative of this was the need to use an arbitrary datum of no more than 14 days extension in the analysis of variance in order to maintain equality of variances between the data for the three genotypes.

The study was not just designed to confirm genotypic variation in the survival of $M$. persicae on artificial diet, but also to conduct an a priori test of Wool \& van Emden's (1981) prediction that the order in which the genotypes would die out would reflect their degree of insecticide resistance based on their level of production of carboxylesterase enzymes and possession of a target site resistance mechanism.

The prediction was indeed fulfilled, with the survival time of the susceptible genotype 4106A being greater than that of any of the resistant clones with elevated carboxylesterase. This might be taken as evidence that having elevated carboxylesterase, which in $\mathrm{R}_{3}$ aphids account for ca 3\% of total body protein (Devonshire \& Moores, 1982), may impose a fitness cost which in turn makes aphids less able to survive on artificial diet. However, since both 800F and 794J produce equivalent levels of carboxylesterase it might be predicted that they would have similar survival rates. This was clearly not the case, with $800 \mathrm{~F}$ surviving significantly longer than 794J. If the survival rates result from direct costs of carboxylesterase 
resistance, then there are two possible explanations. The first is that the presence of a target site mutation (kdr), in the homozygous state, alongside elevated carboxylesterase in 794J makes it less able to survive on the artificial diet. Alternatively, the presence of the major chromosomal abnormality (A1-3 translocation) in 794J, which is always associated with elevated E4, might make the aphids less likely to survive under stress, possibly through some form of disruption to their genome. However, either case suggests that it is not the level of carboxylesterase enzyme per se that determines survival (if it were, then the survival of $800 \mathrm{~F}$ and 794J should have been the same), but the presence of another linked factor. If this is correct then it would be expected that the revertant 794Jrev clone which also has the target site kdr resistance, the same chromosomal translocation and the same E4 gene amplification, but does not make elevated esterase, would survive to the same extent as $794 \mathrm{~J}$. This is indeed the case for the limited data available and the same lack of difference (in survival) between three $\mathrm{R}_{3}$ clones and three clones that had reverted from $\mathrm{R}_{3}$ has also been reported in $M$. persicae overwintering as active forms in the field (Foster et al., 1996) and moving at low temperatures from deteriorating leaves in the field and the laboratory (Foster et al., 1997).

The fully insecticide-susceptible clone (4106A) showed a potential to survive on the artificial diet that was clearly qualitatively and not just quantitatively different from that of the other genotypes. Just the same kind of qualitative difference was shown in Wool \& van Emden's (1981) study of another highly organophosphate-susceptible genotype from a quite different source more than 30 years ago. We lay emphasis on this qualitative result because it is this that enables us to claim that the difference between 4016A and the two insecticideresistant genotypes is outside the range of variation that could be expected if the order in which the three genotypes survived on artificial diet was no more than the 1 in 6 chance of a particular arrangement of three genotypes. 
In view of the observation by Foster et al. $(1996,1997)$ that insecticide-resistant genotypes of $M$. persicae were reluctant to leave deteriorating leaves, it seemed possible that the requirement of aphids in our experiments to switch between sachets at diet changes might introduce an artefact. Thus, differential survival times of genotypes might merely reflect their ability to cope with an unnatural element of the artificial diet procedure rather than the diet per se. However, the counts of aphids that failed to switch sachets at diet changes were very similar in the three genotypes monitored (Table 1). Because the number of aphids on the diet was not always the same for each genotype, it might be argued that the percentage of less mobile aphids is more important than the number and that this percentage might be higher in the insecticide-resistant genotypes, leading to their early extinction. However, most of the data on aphids remaining on the punctured sachets were intentionally taken early on in the progress of the culture. After starting replicates, numbers in all diet tubes rose rapidly and, even with $800 \mathrm{~F}$ and $794 \mathrm{~J}$, were soon too great to count with any accuracy. Even if a pessimistic estimate of 100 aphids per diet tube is used, it is clear that the $\pm 6-7 \%$ of less mobile aphids did not prevent rapid population increase with any of the genotypes.

The different survival times on artificial diet probably partly reflect differences in the rate of reproduction of the genotypes. Fenton et al. (2010b) measured the size of populations on four host plants (two Brassicaceae and two Solanaceae) 15 days after inoculation with one individual of each of 18 clonal lineages of $M$. persicae. There were statistically significant differences at $P<0.001$ between both host plants and lineages, and the lack of interaction between these two factors showed that the ranking of reproductive performance for individual genotypes was genotype-specific. Included in Fenton et al.'s experiment were two of the three genotypes used in our study, and their results are comparable with ours in that for 794J aphid numbers were only about half those of 4106A (except on radish where numbers were not very different). We assume that, after only 15 days, numbers were still rising, whereas our 
experiments ran to extinction. The telescoping of aphid generations within the mother allowed by parthenogenesis and viviparity means that the initial performance of the aphids on transfer to diet will be affected by the mothers' diet (Adams \& van Emden, 1972). Thus the initial burst of reproduction on diet observed for all genotypes at least partially reflects the high quality of Chinese cabbage as the host plant common for all genotypes before transfer. Subsequent relatively sudden declines therefore demonstrated a secession in recruitment, and showed that differences in development time and fecundity cannot be responsible for much of the observed inter-genotypic variations in survival time. Our experiments also differ from those of Fenton et al. (2010b) in that the diet is devoid of the allelochemical challenge, but it is clearly nutritionally unsatisfactory. In general, total fecundity of $M$. persicae on the artificial diet is only about one-third of that achieved on plants (van Emden, 2009). It would be interesting to repeat our experiments with a different stress factor such as a plant species known to be a poor host of $M$. persicae.

The low frequency of insecticide-resistant genotypes in pest populations before insecticides are applied suggests that they are usually at a disadvantage. It is therefore not surprising that such genotypes cope less well with the stress of a simplified artificial diet. However, the association of insecticide-resistance and reduced fitness is not in itself any evidence of cause and effect. Phenomena such as lower fecundity clearly could be a direct cost of the metabolic resistance mechanism, e.g. the synthesis of a detoxifying enzyme, which has been shown experimentally with aphids (see Introduction). Other examples of reduced fitness of insecticide-resistant genotypes that have been demonstrated with $M$. persicae, such as reluctance to move from deteriorating leaves (Foster et al., 1996, 1997), reduced winter survival (Foster et al., 1996) and greater vulnerability to parasitoid attack (Foster et al, 2007), are somewhat less likely to represent the energetic cost of an increase in the synthesis of carboxylesterases. By contrast, although only two results for the revertant 
strain 749Jrev (which is genetically identical to 749J but does not produce elevated carboxylesterase levels) could be obtained before it died out at Rothamsted, we can be confident that the short survival on diet of $749 \mathrm{~J}$ is not a fitness cost of over-producing carboxylesterase. Fig. 2 shows that, in the replicate started on 6 November 2006, the survival of 794Jrev on that occasion was almost identical to that of either the original $794 \mathrm{~J}$ or the other R3 carboxylesterase-resistant genotype 800F. Yet more convincingly, 794Jrev showed even poorer survival than either 794J or $800 \mathrm{~F}$ in the replicate started on 21 January 2009. Thus we believe the different survival times on our artificial diet of the three $M$. persicae genotypes $4106 \mathrm{~A}, 800 \mathrm{~F}$ and $794 \mathrm{~J}$ are not related to the costs of the associated carboxylesterase production. However, we cannot rule out other costs of the chromosomal translocation or of other insecticide-resistance mechanisms such as the kdr mechanism in clone 749J. The different survival times may even reflect differences in the biochemical metabolic capabilities of the genotypes or even of their endosymbionts. In describing the survival on the artificial diet of M. persicae for over ten years at Reading, van Emden (1988) wrote "It is nevertheless likely that we have not so much developed a diet-rearing method suitable for $M$. persicae, as by chance found a $M$. persicae suitable for our diet-rearing method". This is perhaps confirmed by the fact that the genotypes from Rothamsted differ from each other in their ability to cope with the artificial diet on which the Reading genotype succeeded so much better than any of them. The Reading aphids were collected over 30 years ago when the composition of $M$. persicae genotypes in the UK is most likely to have been rather different to that which prevails today, at least in part due to the changes in insecticide modes of action used to control this pest.

We conclude that genotypes of M. persicae which have low fitness in the field may often also show poor performance on artificial diet. If they carry an insecticide-resistance mechanism, their gene frequency will increase when the relevant insecticide is applied, 
whereas that of other genotypes of low fitness will not. This will result in an apparent correlation between low fitness and insecticide resistance which may or may not, however, involve cause and effect.

Overall, although we have seen differential survival rates of the aphid clones, all the genotypes showed good initial reproduction on the diet, thriving for several weeks before declining. This is long enough to make the diet useful for many purposes, especially for the bioassay of toxic/attractant/deterrent compounds (van Emden, 2009). Nevertheless, unless only one particular genotype of $M$. persicae is of interest, it would clearly pay to use an insecticide-susceptible genotype in such experiments.

A further point to consider is the very variable combined performance on artificial diet of all three Rothamsted $M$. persicae genotypes in the different replicates. The most likely explanation is that the artificial diet represented a considerable stress on all of them, and that storage of the diet (which is not sterilised before the sachets are prepared) even at $-20^{\circ} \mathrm{C}$ leads to progressive slight deterioration with which the Reading insecticide-susceptible genotype which lasted so long on diet could cope far better than can other $M$. persicae genotypes. Recent screening using micro-satellite genotype analysis suggests that the UK $M$. persicae population consists at any one time of a very limited number of predominant asexual clones (Fenton et al., 2010a). It would be interesting to see how these fare on artificial diet versus an insecticide-susceptible (wild type) clone.

In conclusion, although our main analysis is restricted to one insecticide-susceptible and two resistant genotypes whose resistance mechanisms are not identical, there is a clear connection (though not necessarily through cause and effect) between insecticide resistance and poor survival on diet. We base this conclusion on three considerations: 
1) A replicated experiment, designed for the purpose, has given the same result as obtained over 30 years ago by a posteriori inspection of their data by Wool \& van Emden (1981) with insecticide-resistant genotypes having elevated carboxylesterase.

2) The survival of the susceptible genotype differed from that of the resistant genotypes not only quantitatively, but also qualitatively in that cultures in most replicates were still thriving when the resistant populations became extinct (Fig. 2). We had to put an artificial limit on the survival time analysed for the susceptible genotype to enable parametric statistical analysis to be used.

3) Since the definition of an insecticide-resistant genotype is that its gene frequency increases from a low level only after application of insecticide, it seems implicit that it must have low fitness in the absence of such application.

\section{References}

Adams, J.B. \& van Emden, H.F. (1972) The biological properties of aphids and their host plant relationships. Aphid Technology (ed. by H. F. van Emden), pp. 47-104. Academic Press, U.K.

Blackman, R.L. (1971) Variation in the photoperiodic response within natural populations of Myzus persicae (Sulzer). Bulletin of Entomological Research, 62, 281-294.

Castañeda, L.E., Barrientos, K., Cortes, P.A., Figueroa, C.C., Fuentes-Contreras, E., LunaRudloff, M.L., Silva, A.X. \& Bacigalupe, L.D. (2011) Evaluating reproductive fitness and metabolic costs for insecticide resistance in Myzus persicae from Chile. Physiological Entomology, 36, 253-260.

Crow, J.F. (1957) Genetics of resistance to chemicals. Annual Review of Entomology, 2, 227 246. 
Devonshire, A.L. \& Moores, G.D. (1982) A carboxylesterase with broad substrate specificity causes organophosphorus, carbamate and pyrethroid resistance in peach-potato aphids (Myzus persicae). Pesticide Biochemistry and Physiology, 18, 235-246.

Eggers-Schumacher, H.A. (1983) A comparison of the reproductive performance of insecticideresistant and susceptible clones of Myzus persicae. Entomologia Experimentalis et Applicata, 34, 301-307.

van Emden, H.F. (1977) Failure of the aphid, Myzus persicae, to compensate for poor diet during early growth. Physiological Entomology, 2, 53-58.

van Emden, H.F. (1988) The peach-potato aphid Myzus persicae (Sulzer) (Hemiptera: Aphididae) - more than a decade on a fully-defined chemical diet. Entomologist, 107, 410.

van Emden, H.F. (2009) Artificial diet for aphids - thirty years' experience. Redia, 2, 163167.

Fenton, B., Margaritopoulos, J.T., Malloch, G.L. \& Foster, S.P. (2010a) Micro-evolutionary change in relation to insecticide resistance in the peach-potato aphid, Myzus persicae. Ecological Entomology, 35, 131-146.

Fenton, B., Kasprowicz, L., Malloch, G. \& Pickup, J. (2010b) Reproductive performance of asexual clones of the peach-potato aphid, (Myzus persicae, Homoptera:Aphididae), colonising Scotland in relation to host plant and field ecology. Bulletin of Entomological Research, 100, 451-460.

Foster, S.P., Harrington, R., Devonshire, A.L., Denholm, I., Devine, G.J., Kenward,. M.G. \& Bale ,J.S. (1996) Comparative survival of insecticide-susceptible and resistant peachpotato aphids, Myzus persicae (Sulzer) (Hemiptera: Aphididae), in low temperature field trials. Bulletin of Entomological Research, 86, 17-27. 
Foster, S,P, Harrington, R., Devonshire, A.L., Denholm, I., Clark, S.J. \& Mugglestone, M.A. (1997) Evidence for a possible fitness trade-off between insecticide resistance and the low temperature movement that is essential for survival of UK populations of Myzus persicae (Hemiptera: Aphididae). Bulletin of Entomological Research, 87, 573-579.

Foster, S.P., Denholm, I. \& Devonshire, A.L. (2000) The ups and downs of insecticide resistance in peach-potato aphids (Myzus persicae) in the UK. Crop Protection, 19, 873879.

Foster, S.P., Tomiczek, M., Thompson, R., Denholm, I., Poppy, G., Kraaijeveld, A.R. \& Powell, W. (2007) Behavioural side-effects of insecticide resistance in aphids increase their vulnerability to parasitoid attack. Animal Behaviour, 74, 621-632.

Hampson, M.J. \& Madge, S. (1986) Morphometric variation between clones of the damsonhop aphid, Phorodon humuli (Schrank) (Hemiptera: Aphididae). Agriculture Ecosystems and Environment, 16, 255-264.

Hampson, M.J. \& Madge, D.S. (1987) Reproduction rates of insecticide-resistant and susceptible strains of the damson-hop aphid, Phorodon humuli (Hemiptera, Aphididae). Acta Entomologica Bohemoslovaka, 84, 181-184.

Kliot, A. \& Ghanim, M. (2012) Fitness costs associated with insecticide resistance. Pest Management Science, 11, 1431-1437.

Lorriman, F. \& Llewellyn, M. (1983) The growth and reproduction of hop aphid (Phorodon humuli) biotypes resistant and susceptible to insecticides. Acta Entomologica Bohemoslovaka, 80, 87-95.

Mittler, T.E. \& Dadd, R.H. (1962) Artificial feeding and rearing of the aphid, Myzus persicae (Sulzer) on a completely defined synthetic diet. Nature, 195, 404.

Needham, P.H. \& Sawicki, R.M. (1971) Diagnosis of resistance to insecticides in Myzus persicae. Nature, 230, 125-126. 
Rider, S.D. \& Wilde, G.E. (1998) Variation in fecundity and sexual morph production among insecticide-resistant clones of the aphid Schizaphis graminum (Homoptera: Aphididae). Journal of Economic Entomology, 91, 388-391.

Stone, B.S., Shufran, R.A. \& Wilde, G.E. (2000) Life history study of multiple clones of insecticide resistant and susceptible greenbug Schizaphis graminum (Homoptera: Aphididae). Journal of Economic Entomology, 93, 971-974.

Wool, D. \& van Emden, H.F. (1981) A possible genetic component in the adaptability of Myzus persicae (Sulzer) clones (Hom. Aphididae) to synthetic diet. Zeitschrift für Angewandte Entomologie, 91, 225-231.

\section{Legends for figures}

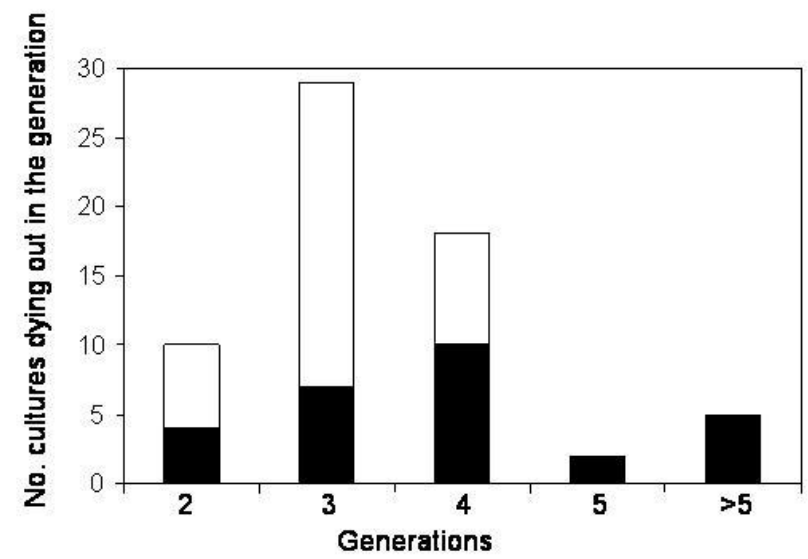

Figure 1. Number of aphid cultures of low (black) and extreme esterase (white) dying out at each generation.

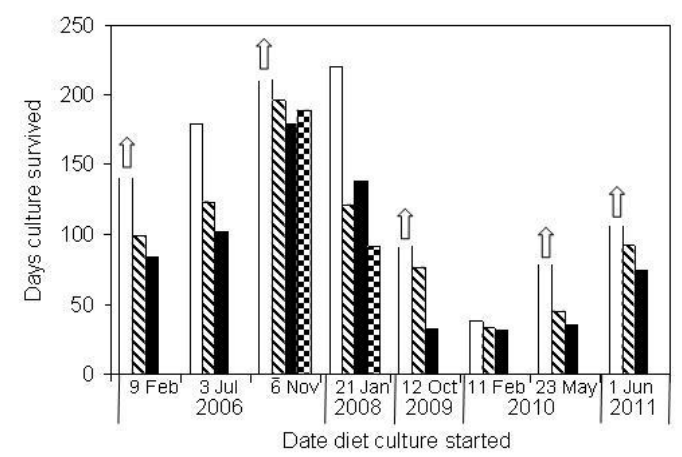


Figure 2. Survival (days) of the different aphid cultures on artificial diet. White columns, 4106A (with top of column open if recording was terminated before extinction); hatched, 800F; black, 794J 
Table 1. Data on survival of three genotypes of Myzus persicae on artificial diet and the numbers remaining on punctured sachets after diet changes.

\begin{tabular}{lcccc}
\hline \multicolumn{1}{c}{ Genotype: } & $4106 \mathrm{~A}$ & $800 \mathrm{~F}$ & $794 \mathrm{~J}$ & $\begin{array}{c}\text { LSD } \\
(P=0.05)\end{array}$ \\
\hline Mean days of survival on diet $(\mathrm{n}=8)$ & $113.13^{1} \mathrm{c}$ & $98.13 \mathrm{~b}$ & $84.38 \mathrm{a}$ & 10.57 \\
& 19.18 & 18.07 & 19.02 & \\
s.e. & $22.08 \mathrm{a}$ & $18.33 \mathrm{a}$ & $19.25 \mathrm{a}$ & 17.83 \\
\hline $\begin{array}{l}\text { Mean no. aphids/3 diet changes } \\
\text { remaining on old punctured diet sachet } \\
\text { (n= 12) }\end{array}$ & & & & \\
\begin{tabular}{l} 
s.e. \\
\hline
\end{tabular} & 6.2 & 6.44 & 6.07 & \\
\hline
\end{tabular}
${ }^{1}$ Maximum datum for survival duration included in analysis for genotype $4106 \mathrm{~A}$ is longer
duration on the two other genotypes +14 days (see text).

Different letters after means within rows indicate significant differences at $P<0.05$. 